Missed Diagnosis

\title{
Foreign body aspiration in the adult: an occult cause of chronic pulmonary symptoms
}

\author{
Issahar Ben-Dov' and Yossef Aelony ${ }^{2}$
}

'Department of Medicine, Hadassah Hebrew University, Mount Scopus, Jerusalem, Israel and ${ }^{2}$ Southern California Permanente Medical Group, Harbor City, California, USA.

\begin{abstract}
Summary: We describe two otherwise healthy subjects with many years sequelae due to lung aspiration. In both, diagnosis was delayed due to lack of history of aspiration. Recurrent pneumonia in the same segment in one, and migratory pneumonia in the other, could have suggested the diagnosis.

Review of the literature shows that occult foreign body aspiration poses difficulties in diagnosis and that often a history of aspiration is lacking. However, the recurrent nature and the localization of the pneumonia, as well as the findings during bronchoscopy, should alert the physician to the possibility of foreign body aspiration.
\end{abstract}

\section{Imtroduction}

Children and adults with certain predisposing factors, are prone to aspiration of foreign bodies. ${ }^{1,2}$ Moreover, since aspiration is usually a stressful experience, an alert adult would be expected to volunteer a clear history of the episode. We recently took care of two patients with long standing pulmonary symptoms. In both cases, foreign body aspiration many years previously was eventually found. Review of previous cases revealed that at presentation patients commonly are not aware of the past aspiration. We describe our cases and review the natural course and clues that should alert the physician to the possibility of foreign body aspiration. ${ }^{3-7}$

\section{Case reports}

Case 1

A 70 year old non-alcoholic man was admitted for recurrent pneumonia during the previous 6 months. He had been a heavy smoker until 9 years previously. Six months prior to admission he had pneumonia which was localized to the anterior segment of the right upper lobe (Figure 1), and resolved with ampicillin. Residual cough persisted. Six months later his

Correspondence: I. Ben-Dov; Department of Medicine, Hadassah University Hospital, Mount Scopus, P.O.B. 24035, Jerusalem 91240, Israel.

Accepted: 13 December 1988 temperature rose again to $39^{\circ} \mathrm{C}$. This time there was no improvement following a course of an oral cephalosporin, and he lost $5 \mathrm{~kg}$ in weight.

Chest X-ray showed free fluid and right basilar segmental infiltrate. Blood and sputum cultures and sputum cytology were repeatedly negative.

Based on the heavy smoking history, persistent lung infiltrates, and weight loss, bronchogenic carcinoma was suspected. Fibreoptic bronchoscopy showed mild inflammatory changes and purulent discharge in the right lower lobe airways, but there was no apparent endo-bronchial lesion. Following the procedure the patient experienced paroxysms of coughing in which a $4 \times 6 \mathrm{~mm}$ coffee bean was expectorated. The patient then volunteered the history that since he had stopped smoking he had adopted the habit of sucking coffee beans, but he could not recall an episode of aspiration. Following the expectoration the symptoms subsided except for an occasional productive cough. Follow-up $\mathrm{X}$-rays showed findings consistent with bronchiectasis.

\section{Case 2}

A 57 year old non-alcoholic man was admitted in September 1984 for evaluation of recurrent right lower lobe pneumonia. He had been well until 1967, when he was hospitalized for a right lower lobe pneumonia, associated with pleural effusion, which was slow to resolve. Rigid bronchoscopy revealed acute inflammatory changes in the right lower lobe airways. 


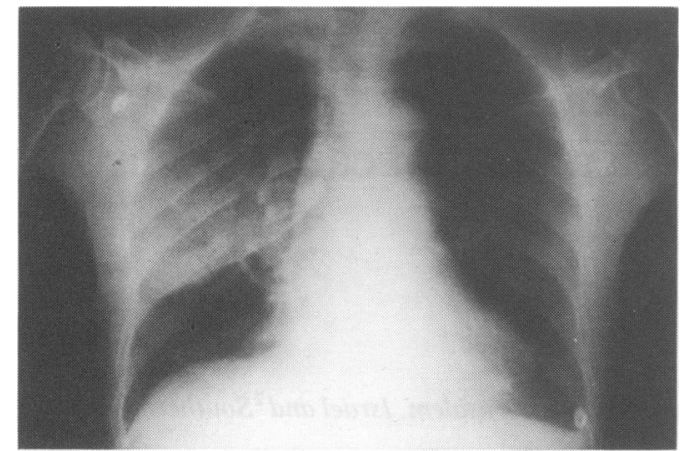

Figure 1 Case 1: chest radiology (PA) taken during a pneumonic episode 6 months before admission, showing opacity localized to the anterior segment of the right upper lobe.

Skin tests for tuberculosis and coccidioidomycosis were positive, but sputum and bronchoscopic cultures were negative, as were cytological smears. The patient gave up smoking during this acute illness.

He subsequently felt well until 1979 , when seen for cough, purulent sputum, and a right lower lobe infiltrate, which responded to antibiotics. Similar episodes occurred annually, all involving the same basilar segment. He then admitted to a chronic productive cough and occasional haemoptysis. Fibreoptic bronchoscopy in June 1984 revealed an oedematous anterobasilar segment containing a densely blackened area, the nature of which was difficult to characterize. Bronchial biopsies showed chronic inflammation of the submucosa and squamous metaplasia. He then felt well until cough and purulent sputum recurred in September 1984. Repeat flexible bronchoscopy revealed a black foreign body in the anterobasilar segment, which was removed with the rigid bronchoscope. When shown to the patient, he recalled aspirating a pumpkin seed prior to his 1967 illness. During 4 years of follow-up he has had no further pulmonary infections.

\section{Discussion}

The two subjects described here were both alert, otherwise healthy, adults who presented with chronic recurrent pulmonary symptoms and required repeated hospitalizations and diagnostic procedures. Bronchial obstruction by an inhaled foreign body was proven to be the cause in each case. This diagnosis had not been considered initially, due to the failure to elicit a history of aspiration, until after the patient witnessed the foreign body being expectorated. In retrospect the migratory nature of the infiltrate in segments not sharing a common airway (case 1), and the repeated infiltrate in the same segment (case 2) could have suggested the diagnosis.

Pulmonary aspiration is expected in adults who share clear predisposing factors which interfere with the normal defense mechanisms protecting the air- $C$. ways. ${ }^{8,9}$ Our cases and others ${ }^{3-7}$ indicate that normal $\vec{F}$ adults are also occasionally subject to aspiration and $\stackrel{\text { ? }}{7}$ its chronic sequelae. In some, the episode occurs? during periods of diminished alertness, such as during $\frac{\overline{\bar{N}}}{\bar{\rho}}$. an accident ${ }^{6}$ or dental extraction. ${ }^{3}$ Others may aspirate $\frac{}{7}$ in a particle-rich environment, such as carpentry, ${ }^{4} \stackrel{\Phi}{\varrho}$ whilst others aspirate during eating with no apparent cause ${ }^{3,4,7}$ Rarely, aspiration occurs during childhood $\rightarrow$ while the sequelae appear or persist into adulthood. ${ }^{7}$ 을

If the foreign body is small, it can lodge in a $\vec{\omega}$ peripheral airway, commonly in the right lower lobe. ${ }^{7 /}$ However, any segment can be involved. ${ }^{5,7}$ Patientso usually experience choking and cough initially, but 3 . later the irritation ceases ${ }^{1,4,5}$ and a symptom-free of period follows which can last from hours to months or even years, the longest recorded being 25 years. ${ }^{4,9,10}$ In a series of 16 adults with foreign body aspiration $\tilde{C}_{0}$ pneumonias, five volunteered a history, five could 6 recall it only after the diagnosis had been made, and six 을 gave no history of aspiration. ${ }^{3}$ Thus, even in a healthy adult, the lack of suggestive history does not rule out aspiration. During the late symptomatic phase, patients may present with bouts of fever, cough, $\vec{\bullet}$ expectoration, haemoptysis (sometimes massive), ${ }_{0}^{3}, 0$ and pleuritic chest pain. ${ }^{8}$ Chest X-ray will only show. the foreign body if it is radiopaque and of sufficie 1 i size (unfortunately most are neither). If an infiltrate is slow to resolve, if it recurs in the same location, ${ }^{3}$ or even if it migrates from one lung to the other or: between segments not sharing a common bronchus $\varnothing$ (our case 1 and reference 6 ), a history suggestive of $\overrightarrow{\overrightarrow{0}}$ foreign body aspiration should be sought carefully. 3 Further radiological evaluation is often unrevealing, but computerized tomography was diagnostic in three? out of four children when specifically looked for ${ }^{13} \bar{O}$ Bronchoscopy is often diagnostic, unless the foreign 3 . body is peripheral, or is surrounded by inflammatory $\overline{0}$ tissue. Thus friable granulation tissue with tumourlike appearance, which shows on histology only $\delta$ inflammatory reaction, or finding a meat fibre ${ }^{15}$ or $₹$ bone, ${ }^{4}$ should suggest aspiration. ${ }^{3,7}$ However, as 윽 shown, even repeated negative bronchoscopic $D$ examinations in a healthy adult do not rule out foreign body.

Pulmonary complications that have been described $\mathscr{N}$ with foreign body aspiration are recurrent and mig- $N$ ratory pneumonia (our case 1 and references 3 and 6 ), $\mathbb{\omega}^{2}$ lung abscess, cysts and cavities, ${ }^{7,8,11}$ asthma-like symp- -0 toms, ${ }^{16}$ bronchiectasis and bronchial stenosis, ${ }^{2,7} 0$ granulomatous process, ${ }^{17}$ bronchiolitis obliterans, ${ }^{18} \frac{C}{D}$

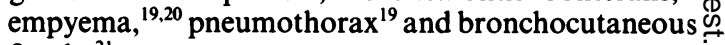
fistula. $^{21}$ 
The definitive treatment for foreign body aspiration is its removal. If performed before permanent damage occurs, the prognosis is good, but bronchiectasis may result and persist. ${ }^{11}$ Usually the rigid bronchoscope should be used, although the role of the flexible fibreoptic instrument is evolving. ${ }^{4.11}$

\section{References}

1. Teig, E. \& Grounds, T.E. Foreign bodies in the lower respiratory tract. Acta Otolaryngol 1967, 224 (suppl): 429-430.

2. Abdulmajid, O.A., Abdelmomen, M.E., Mohamed, M.M. \& Ibrahim, S.K. Aspirated foreign bodies in the tracheobronchial tree: report of 250 cases. Thorax 1976, 31: $635-640$.

3. Linton, J.S.A. Long-standing intrabronchial foreign bodies. Thorax 1957, 12: 164-170.

4. Wolkove, N., Kreisman, H., Cohen, C. \& Frank, H. Occult foreign-body aspiration in adults. JAMA 1982, 248: $1350-1352$.

5. Chopra, S., Simmons, D.H., Cassan, S.M., Becker, S. \& Ben-Isaac, E. Bronchial obstruction by incorporation of aspirated vegetable material in the bronchial wall. $\mathrm{Am}$ Rev Respir Dis 1975, 112: 717-720.

6. Hargis, J.L., Hiller, F.C. \& Bone, R.C. Migratory pulmonary infiltrates secondary to aspirated foreign body. JAMA 1978, 240: 240.

7. Denney, M.K., Berkas, E.M., Snider, T.H. \& Nedwicki, E.G. Foreign body bronchiectasis. Dis Chest, 1968, 53: 613-616.

8. Newman, G.E., Effman, E.L. \& Putman, C.E. Pulmonary aspiration complex in adults. Curr Probl Diagn Radiol 1982-3, 11-12: 3-46.

9. Barlett, J.G. \& Gorbach, S.L. The triple threat of aspiration pneumonia. Chest 1975, 68: 560-566.

10. Da' Silva, A.M.M. Foreign body aspirations: case reports. S Afr Med J 1982, 61: 130-138.

11. Editorial. Inhaled foreign bodies. $\mathrm{Br}$ Med J 1981, 282: $1649-1650$.
In summary, fully alert adults are occasionally subject to foreign body aspiration. A relevant history is commonly lacking and negative bronchoscopy does not rule out the diagnosis. The combination of certain clinical, radiological and bronchoscopic findings should suggest the diagnosis.

12. Leonidas, J.C., Stuber, J.L., Rudavsky, A.Z. \& Abramson, A.L. Radionuclide lung scanning in the diagnosis of endobronchial foreign bodies in children. J Pediatr 1973, 83: 628-631.

13. Berger, P.E., Kuhn, J.P. \& Kuhns, L.R. Computed tomography and the occult tracheobronchial foreign body. Radiology 1980, 134: 133-135.

14. Cotton, E.K., Abrams, G., Vanhoutte, J. \& Burrington, J. Removal of aspirated foriegn bodies by inhalation and postural drainage. A survey of 24 cases. Clin Pediatr 1973, 12: 270-276.

15. Ristagno, R.S., Kornstein, M.J. \& Hansen-Flaschen, J.H. Diagnosis of occult meat aspiration by fiberoptic bronchoscopy. Am J Med 1986, 80: 154-156.

16. Fine, A.J. \& Abram, L.E. Asthma and foreign bodies. Ann Allergy 1971, 29: 217-220.

17. Knoblich, R. Pulmonary granulomatosis caused by vegetable particles. Am Rev Respir Dis 1969, 99: 380-389.

18. Blumgart, H.L. \& MacMahon, H.E. Bronchiolitis fibrosa obliterans: clinical and pathological study. Med Clin North Am 1972, 13: 197-214.

19. Yellin, A., Tomer, A., Zwas, S.T., Hassin, D., Leiberman, Y. \& Bank, H. An unusual case of empyema; intrapleural migration of an inhaled oat head in an adult. Thorac Cardiovasc Surg 1983, 31: 317-319.

20. Ross, D.N. Inhaled intrapleural foreign body, report of a case. Br J Surg 1954, 41: 412-414.

21. Cavens, T.R., McGee, M.D., Miller, R.R. \& Bowen, D.A. Pneumocutaneous fistula secondary to aspiration of grass. J Pediatr 1973, 82: 737-738. 